TRABAJO DE EXTENSIÓN

\title{
HUERTOS FRUTALES AGROECOLÓGICOS Y PREVENCIÓN DE ZOONOSIS COMO ALTERNATIVA PARA LA SEGURIDAD ALIMENTARIA
}

\section{Agroecological fruit plant nurseries and zoonosis prevention as food safety alternative}

\author{
Alayón Luaces, Paula ${ }^{1}$; Benítez, Andrea ${ }^{2}$; Montes de Oca, Amabelia ${ }^{3}$; Gaiad, José E. ${ }^{1}$; \\ Gómez Herrera, Melanie D. ${ }^{1}$; Sugita, Nicolás H. ${ }^{1}$ \\ ${ }^{1}$ Facultad de Ciencias Agrarias y ${ }^{2}$ Facultad de Ciencias Veterinarias de la Universidad Nacional del \\ Nordeste. Sargento Cabral 2131. 3400 Corrientes Argentina; \\ ${ }^{3}$ EFA 141 Fortaleza Campesina. Paraje Buena Vista - Campo Bermejo - Zona Rural General San Mar- \\ tín. Chaco Argentina. \\ E-mail: palayonluaces@yahoo.com
}

\section{RESUMEN}

El proyecto se llevó adelante en la zona de San Martin Chaco, con familias relacionadas a la EFA 141 Fortaleza Campesina, de los niveles secundario y terciario y con propuestas extensivas a la comunidad en general. Participaron docentes y alumnos de tres unidades académicas (FCA y FCV UNNE y EFA Fortaleza Campesina) y colaboraron activamente representantes de la sociedad como el INCUPO y la Municipalidad de General San Martin. El proyecto buscó promover la seguridad alimentaria de la comunidad y el aprovechamiento económico de la producción. Se realizó una programación productiva, integrando el conocimiento por medio del manejo de las especies frutales, del saneamiento de mascotas y animales domésticos, que pudieran alcanzar al sistema productivo y de la concientización de los beneficios nutricionales que estos frutales les ofrece. Se instalaron viveros de frutales no solo para el aprovechamiento directo sino también para el fortalecimiento de lazos entre familias para el intercambio de material de propagación y semillas. Se realizaron encuentros teórico-prácticos formativos, instalación participativa de los huertos y viveros, participación en ferias, prevención y sensibilización de zoonosis y concientización nutricional. El desarrollo de este proyecto ofreció a las familias, pequeños productores y comunidad de San Martin Chaco aspectos prácticos y valiosos de los huertos frutales para fortalecer su seguridad alimentaria. Sumado a ello, en nuestra comunidad Universitaria fue un aporte de experiencias de trabajo en grupos interdisciplinarios que potencia en los alumnos el profesional extensionista con sensibilidad social.

Palabras clave: agroecología; alimentos; diversificación; familias; salud.

\begin{abstract}
The project was carried out in San Martin, Chaco, along with families associated with secondary and tertiary levels from EFA (Family Agricultural School) 141 Fortaleza Campesina and the whole community. Teachers and students from three academic units (FCA and FCV UNNE and EFA 141 Fortaleza Campesina) participated and representatives from INCUPO and General San Martin Town Hall cooperated with them. The project sought food safety for the community and cost-effective use of production. Profitable programming was carried out integrating expertise as regards: fruit varieties management, pets and domestic animals sanitation able to reach the productive system and awareness of local fruit nutritional benefits. Fruit plant nurseries were installed, not only for direct use but also for strengthening ties between families through the exchange of propagation material and seeds. Several events were held such as: theory and practice training sessions, cooperative installation of orchards and nurseries, participation at fairs, prevention and awareness of zoonoses and nutritional understanding. The development of this project offered families, small producers and San Martin Chaco community practical and valuable aspects of fruit orchards to strengthen their food security. Furthermore, to our University community, it was a contribution of experiences in interdisciplinary groups that enhances the extensionist professional work with social sensitivity among students.
\end{abstract}

Key words: agroecology, diversification, families, food, health.

Recibido: 08/jun/2020. Aceptado: 25/jun/2020 
Alayón Luaces, P. et al.: Huertos frutales agroecológicos y prevención de zoonosis.

\section{INTRODUCCIÓN}

La comunidad de pequeños productores agrícolas de General San Martín Chaco se encuentra alrededor de la ciudad y se trata en general, de familias campesinas con bajos ingresos. Los productores con los que se trabajó son de subsistencia, es decir que la mayor parte de lo producido y cosechado por ellos es destinado al autoabastecimiento de las familias que lo produjeron, con poco resto para su comercialización. Si bien las producciones son diversificadas cuentan con poca tecnología, y están implantadas en pequeñas superficies ( 1 o 2 hectáreas $)$ ubicadas en campos fiscales ocupados y sin título de propiedad. La venta de las producciones la realizan en los mercados locales (pueblos y ciudades cercanas) a intermediarios y en algunos casos en las ferias francas de San Martín. Es decir que entre los problemas que conciernen a estas familias se encuentra el escaso capital de trabajo, muy poca superficie de tierra disponible, bajos ingresos anuales y dificultades para generar inversiones. Otro aspecto fundamental en estas comunidades es la problemática sanitaria y el uso de agroquímicos para el manejo de la producción, lo cual muchas veces pone en riesgo la salud de los integrantes de la comunidad, pudiendo provocar distintas enfermedades y potenciales intoxicaciones. Estas situaciones se traducen indefectiblemente en la dificultad para el acceso físico y económico a suficientes alimentos inocuos y nutritivos para satisfacer sus necesidades alimenticias y poder llevar así una vida activa y sana, concepto que se ajusta a la definición de seguridad alimentaria según la FAO (2011). Actualmente la Tecnicatura Superior en Agroecología de la EFA brinda herramientas para que estas familias se interioricen en las prácticas agroecológicas. La complementación de estos recursos aplicados a los huertos frutales permitiría la ampliación de la oferta alimentaria con especies de alto valor nutritivo y comercial.

Varios programas, proyectos e instituciones abordaron la problemática socioeconómica de las familias en la región, entre las que se encuentran: La Feria Franca de San Martín (Programa Social Agropecuario) con grupos de pequeños productores, Programa ProHuerta de la Agencia de Extensión de INTA, acciones de ONGs de promoción y desarrollo como INCUPO y Asociación ENCUENTRO, con un fuerte apoyo a las organizaciones campesinas y la promoción de los derechos ciudadanos.

En la localidad de General San Martín el INCUPO participó en el proceso de expropiación de 400 hectáreas que se destinaron a 30 familias que vivían al borde de la ruta y que hoy están asentadas en el lugar, haciendo un proceso de arraigo en la tierra conseguida con acciones organizativas de las familias junto con organizaciones locales e instituciones.

Las enfermedades zoonóticas son un grupo de enfermedades infecciosas que se transmiten de forma natural de los animales a los seres humanos, por lo que reducir los riesgos de transmisión de zoonosis al ser humano a través del consumo de productos de origen animal es clave en la seguridad alimentaria de la comunidad.

Se consideró que la situación de la comunidad favorece la propuesta de ampliación de la oferta alimentaria con especies de alto valor nutritivo y comercial, como lo son los frutales. Atendiendo al concepto «Una sola salud», de que la salud humana y la sanidad animal son interdependientes y están vinculadas a los ecosistemas en los cuales coexisten, la instalación de huertas frutales agroecológicas y la prevención de la zoonosis, surgieron como posibilidad para mejorar la calidad alimentaria familiar y comunitaria, además de generar sustento y obtener ingresos.

El proyecto propuso el desarrollo de huertos de frutales en San Martín Chaco con participación de los dos niveles de la EFA Fortaleza Campesina, INCUPO, docentes y estudiantes de dos unidades académicas de la UNNE (FCA y FCV), Municipalidad de San Martín en busca de la diversificación frutícola para la seguridad alimentaria en el marco de la producción agroecológica. La aplicación de este proyecto involucra aspectos relacionados directamente a la seguridad alimentaria de la comunidad y a la posibilidad del aprovechamiento económico y social de la producción.

\section{OBJETIVOS}

\section{Objetivo General}

Promover la implantación de huertos frutales de especies diversas y capacitar en la prevención de zoonosis, para fomentar una alimentación saludable y variada. 


\section{Objetivos Específicos}

1. Fomentar y complementar la alimentación saludable a través de la ejecución participativa de huertos y viveros frutales en terrenos de la EFA y núcleo familiar de alumnos.

2. Capacitar a las familias, docentes y alumnos de la EFA en la propagación, aplicación de prácticas de manejo en frutales agroecológicos y beneficios alimentarios de su consumo.

3. Promover y sensibilizar en la prevención de la zoonosis, en el marco de salud alimentaria.

4. Fomentar en los estudiantes el compromiso social para su formación integral como futuros profesionales potenciando el rol extensionista con sensibilidad social.

5. Mejorar la participación familiar, grupal y comunitaria para potenciar el saber popular en el aprovechamiento del proyecto.

\section{METODOLOGÍA}

Para el desarrollo del proyecto se organizaron diferentes intervenciones:

Relevamiento de situaciones de las familias: en esta primer instancia se realizó un taller de relevamiento de datos bases para utilizarlos para preparar los marcos teórico prácticos de intervención para los siguientes encuentros. Se organizaron grupos de 8-10 participantes autonucleados por temas afines, donde se realizaron preguntas orientativas claves para relevar problemáticas y áreas de interés, las cuales eran volcadas en afiches preparados para tal fin. Cada grupo fue moderado por un alumno universitario participante del proyecto UNNE en el Medio. Luego del trabajo en grupos se realizó una puesta en común con todos los presentes, finalizando con una lista resumen de temas problema a seguir trabajando en futuros encuentros.

Encuentros teórico-prácticos formativos- Transferencia: en base a los temas prioritarios que surgieron del relevamiento inicial se organizaron encuentros teórico - prácticos a fin de trasmitir conceptos de huertos frutales. Se realizaron presentaciones con soportes digitales, se llevaron plantas de frutales, herramientas de trabajo y se realizaron explicaciones de manejo en el marco de la producción agroecológica. Se elaboraron boletines de información técnica digital como material de difusión y apoyo para implementar las prácticas en terreno.

Instalación participativa de huertos frutales y viveros: se fijaron sitios estratégicos para la instalación de huertos frutales modelo y viveros, accesibles a la comunidad educativa de la EFA y a la sociedad en general. La instalación de los lotes se realizó con acciones participativas de la UNNE, alumnos EFA, coparticipantes y destinatarios del proyecto. Los frutales a implantados fueron diversos, acordes a la situación edafoclimática del lugar. Con estas actividades se buscó fortalecer vínculos, crear lazos solidarios y establecer un espacio de intercambio de material.

Prácticas agronómicas a campo: en los lotes de la EFA y en los de productores que tenían plantaciones productivas de frutales fueron capacitados en forma práctica para el mantenimiento y manejo agronómico de las especies existentes en sus lotes.

Sensibilización y prevención de la zoonosis: se capacitó a alumnos y docentes de la EFA y familias en medidas preventivas y culturales, brindando charlas informativas para transmitir medidas de profilaxis tendientes a reducir las posibilidades de contaminación ambiental y concientizar acerca de enfermedades trasmisibles al hombre fomentando, la prevención de las zoonosis. Para el cuidado y prevención de las enfermedades se contó con material para realizar las vacunaciones y aplicaciones de antiparasitarios de amplio espectro para animales coexistentes con las familias, informando además sobre las prácticas de manejo sanitario preventivas de las enfermedades zoonóticas. Así mismo, en estas oportunidades de encuentro participaron agentes sanitarios municipales y productores, quienes oportunamente capitalizaron estas capacitaciones para la implementación en estructuras intermedias locales (Mesa de Certificación de Productos).

Participación en ferias: se participó en el marco de la feria Mi Chacra, feria de pequeños productores agropecuarios donde se llevaron productos de los huertos frutales, subproductos de los mismos y material vegetal para intercambio entre productores. 
Alayón Luaces, P. et al.: Huertos frutales agroecológicos y prevención de zoonosis.

Reuniones periódicas de los integrantes del proyecto: algunas fueron presenciales y otras por medio de medios virtuales, como grupos de WhatsApp, donde se pusieron en valor las informaciones relevadas, tanto por los docentes como por los alumnos, para generar el debate constructivo y ajustar acciones si fuera necesario acompañando a los estudiantes de la UNNE en sus prácticas profesionales.

Difusión: con el fin de invitar a la mayor cantidad de interesados posibles, de transmitir la información generada en las diferentes acciones de este trabajo y facilitar a los todos los actores la posibilidad de disponer de la misma, se realizaron tareas de difusión por varias vías:

- Virtual (grupo WhatsApp, Facebook de la EFA).

- Medios de comunicación radial de la comunidad de San Martín con la colaboración del INCUPO y acciones facilitadas por medio de la Municipalidad.

- Boletines digitales de información técnica, elaborados para cada encuentro teórico práctico como material de difusión y apoyo para implementar las prácticas en terreno.

\section{RESULTADOS}

\section{Actividades que realizaron los estudiantes}

Los estudiantes de las dos Unidades Académicas de la UNNE participaron activamente en las tareas de capacitación tanto teórica como práctica, para la cual fueron previamente instruidos. Estuvieron en contacto con los alumnos, docentes y familias de la EFA facilitando el intercambio de información. Durante el desarrollo de los encuentros y talleres realizaron actividades de asistencia en general y coordinación. El proyecto ofreció un buen escenario para que los estudiantes adquieran destrezas y experiencias útiles en el campo laboral, elementales para su futuro actuar profesional, promoviendo un perfil de profesionales que consideren a la producción y al productor en un todo único. Además, aportaron una amplia experiencia en lo que se refiere al trabajo en grupos interdisciplinarios y potenciar en los alumnos el profesional extensionista con sensibilidad social.

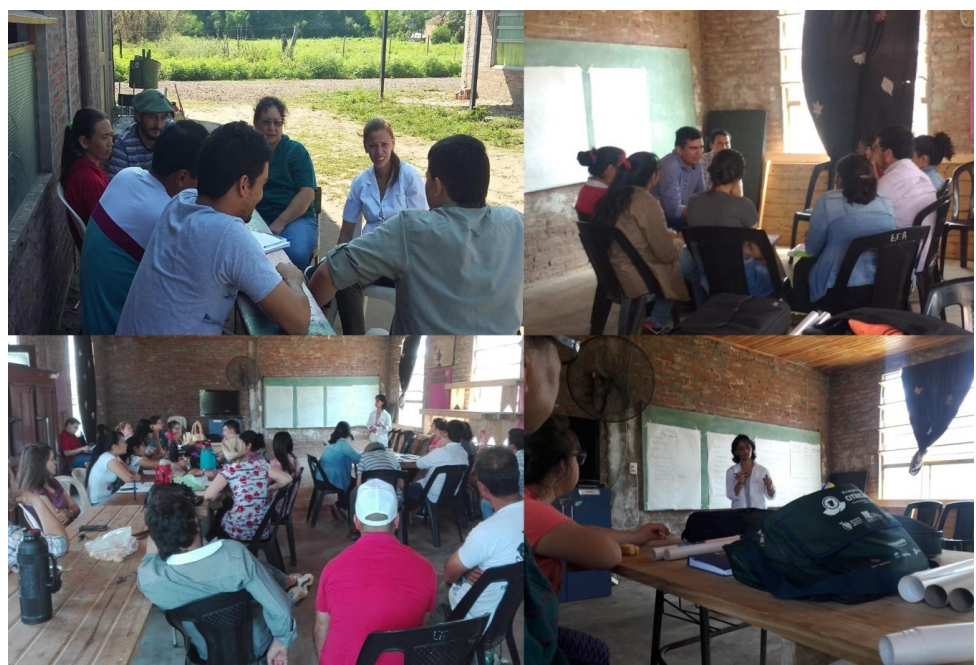

Figura 1. Taller de relevamiento de datos. Intercambio de conocimientos y demanda por parte de las familias y sector productivo de esta comunidad, que permitió detectar situaciones que requirieran atención.

\section{Relevamiento de situaciones de las familias}

Estos encuentros fueron clave para la retroalimentación de los docentes buscando el intercambio de conocimientos y de demanda por parte del sector productivo de esta comunidad, y permitieron detectar situaciones que requieren atención. De este relevamiento surgieron inquietudes en relación a propagación de especies frutales, manejo de vivero, cultivos de palto, mango, mamón, ananá, prácticas de poda y normativa de HLB para cítricos; así como también se hizo visible la fuerte interacción entre las actividades agrícolas y de explotación de animales de consumo, como medio de subsistencia de las familias alcanzadas por el proyecto.

En estas tareas participaron junto con docentes y alumnos de FCA y FCV-UNNE, diferentes actores de la comunidad de San Martín: alumnos y docentes de la EFA, productores y feriantes entre otros (Figura 1). Basado en este relevamiento se realizaron las capacitaciones y transferencias del ciclo de extensión. 


\section{Encuentros teórico-prácticos formativos}

Basado en el relevamiento inicial se abordaron acciones para su mejora productiva: viveros (diversificación de frutales), implantación de nuevos huertos, recuperación de lotes en producción y tareas de prevención de zoonosis en varias especies de granja. Se realizaron seis encuentros formativos que incluyeron los temas propagación de frutales, manejo de vivero, cultivos de palto, mango, mamón, ananá y normativa de HLB para cítricos. Los mismos se realizaron en las instalaciones de la EFA 141 Fortaleza Campesina (Figura 2) y en el Centro Regional de Estudios Superiores (CRES, Figura 3). Asistieron a los mismos alumnos de secundario y terciario de la EFA, pequeños productores, familias, feriantes y público de la comunidad. Esta información sirvió como insumo para realizar acciones en el sector y así consolidar la fruticultura con un enfoque agroecológico en búsqueda de mejorar la oferta alimentaria con especies de alto valor nutritivo y comercial, como lo son los frutales.

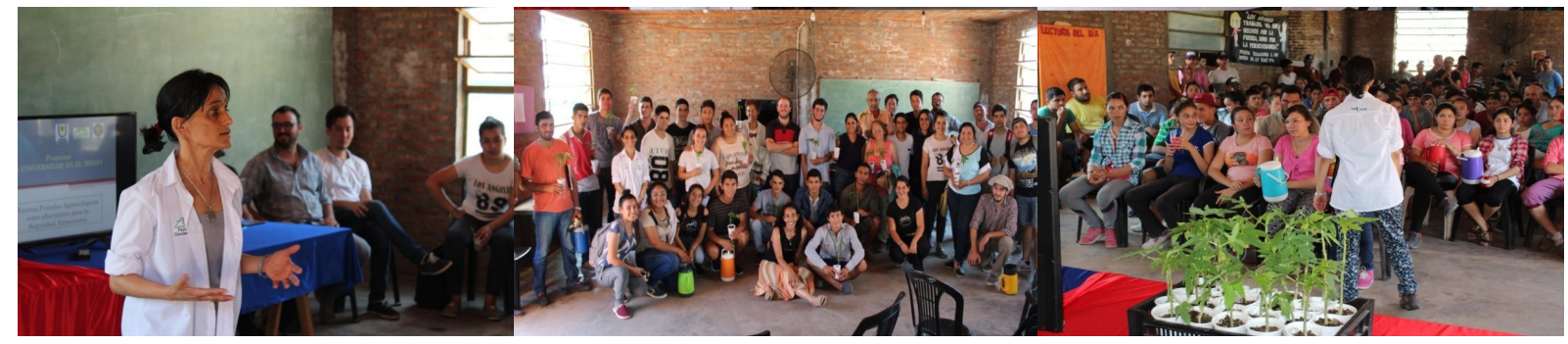

Figura 2. Encuentros teórico-prácticos formativos de transferencia: en base a los temas prioritarios que surgieron del relevamiento inicial. Capacitación cultivo de mamón en la EFA 141 Fortaleza Campesina.

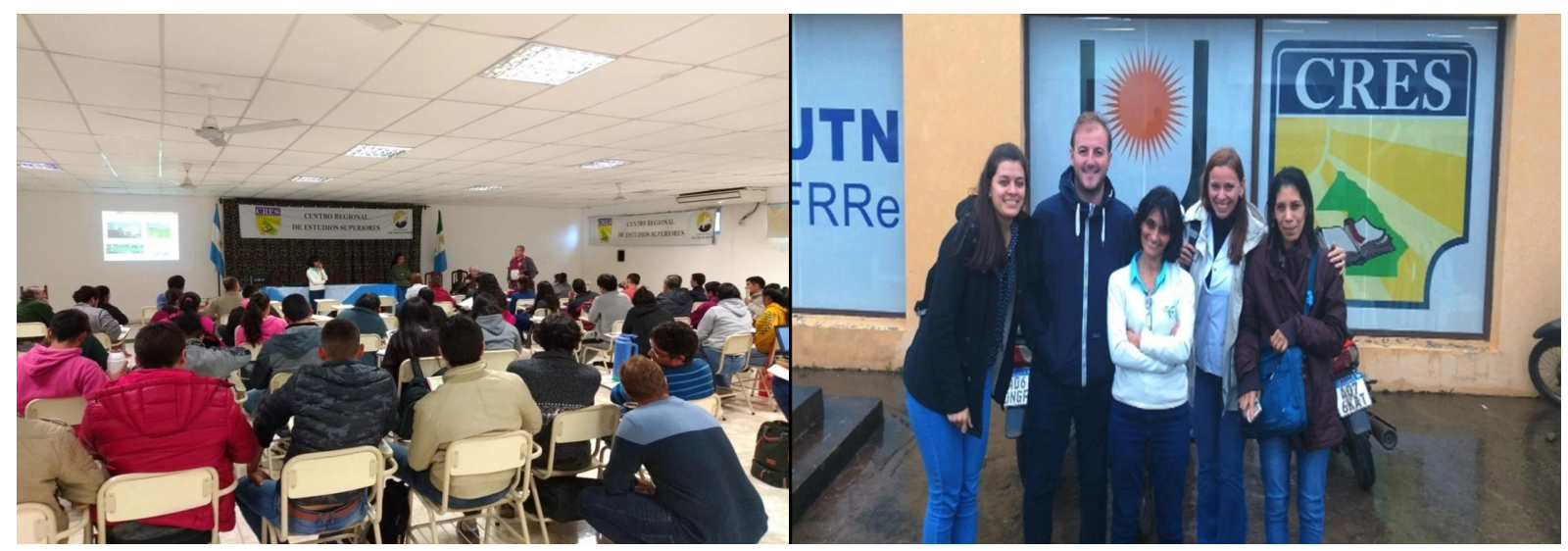

Figura 3. Encuentro teórico-práctico formativo de normativa de HLB en cítricos en el Centro Regional de Estudios Superiores - General San Martín Chaco (CRES).

\section{Instalación participativa de huertos frutales y viveros}

La instalación de viveros fue posible partiendo de material vegetal de frutales que fue facilitado por el programa. Los viveros fueron instalados en la EFA Fortaleza Campesina y en la chacra de un productor modelo (Sr. Soto Marcelo y Estela García: Chacra agroecológica Paraíso en Colonia Lote 90). En el marco de uno de los encuentros formativos se realizaron prácticas para propagar ananá, aguaí, mamón, mango, pitahaya, ñangapirí y guayaba. Este material permitirá el establecimiento de nuevos huertos frutales como recursos de las familias para mejorar su alimentación. Además de los viveros se trabajó en la instalación del huerto frutales de la EFA con colaboración de los alumnos, el cual servirá para prácticas y aplicación de acciones asociadas a los sistemas productivos. En encuentros siguientes se continuó utilizando el espacio como escenario práctico de nuevos temas formativos. También se realizaron observaciones de prácticas de manejo culturizadas en la zona de granja de la EFA 141(Figura 4). 


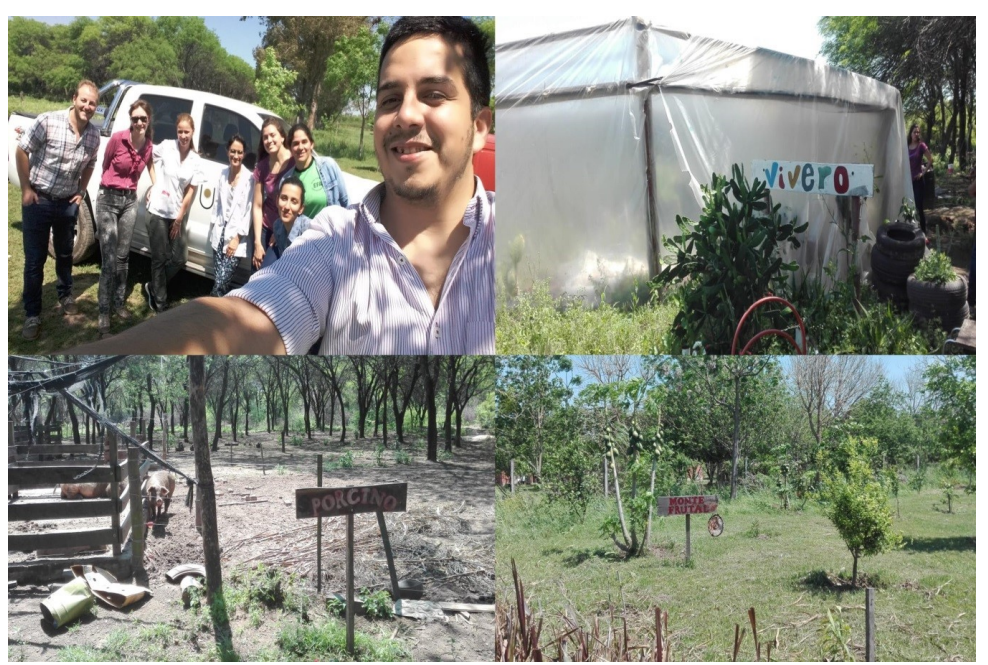

Figura 4. Instalación participativa de viveros y atención de huerto frutal y granja de la EFA 141 Fortaleza Campesina. San Martín - Chaco.

\section{Prácticas agronómicas a campo}

En el lote de frutales a campo que cuenta la EFA y en el campo particular del Sr. González Zarate, Ramón y Telier Nadia A. Lagunas Lobos (Gral. San Martín Chaco); se realizaron prácticas asociadas a la sanidad de los frutales. Se realizaron recorridas con los participantes reconociendo plagas y enfermedades generando debates de propuestas de control con un enfoque agroecológico. Se realizó una capacitación práctica de poda de especies frutales implantadas (vid, cítricos, higo, manzanas, maracuyá). Se mostró el uso de las herramientas apropiadas para cada tipo de corte y las exigencias propias de cada especie (Figura 5).

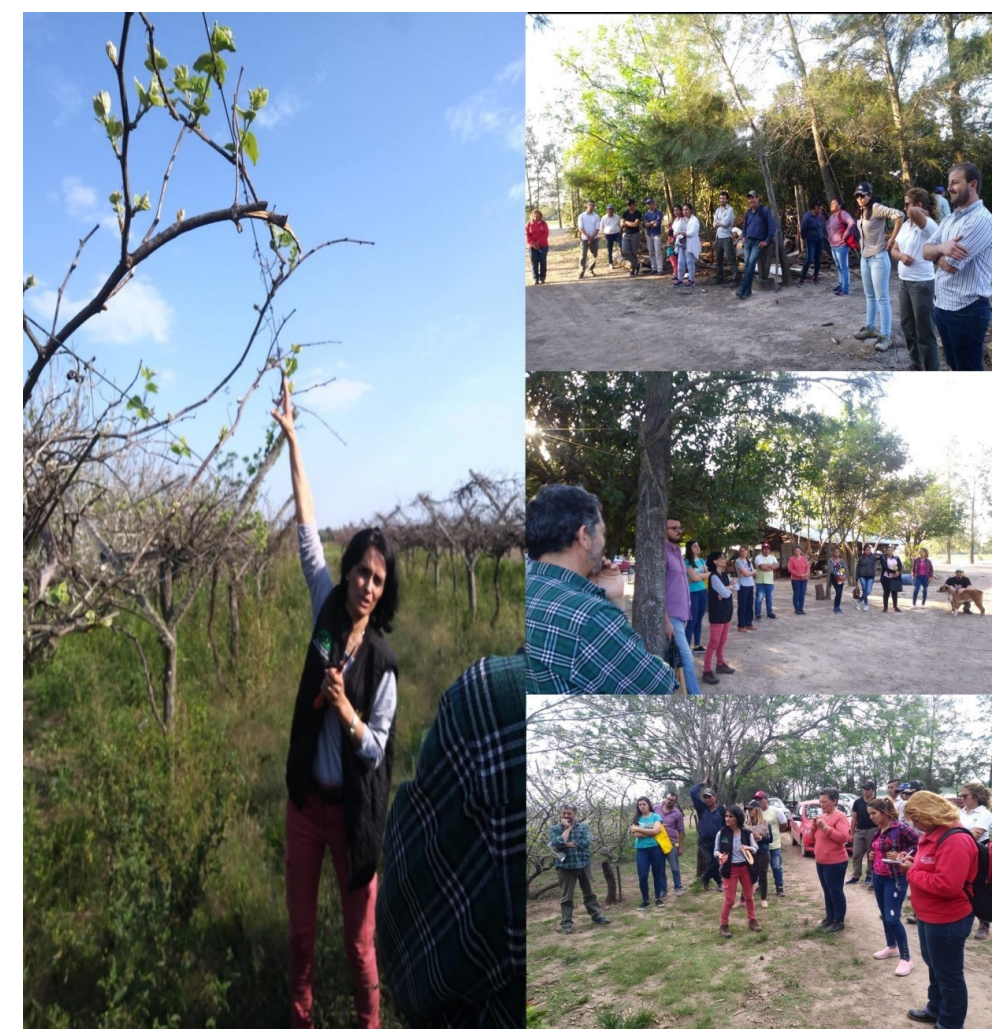

Figura 5. Capacitación práctica de poda de especies frutales implantadas (vid, cítricos, higo) en la finca de productor en Laguna Lobos (Gral. San Martín Chaco). 


\section{Sensibilización y prevención de la zoonosis}

Se propiciaron distintos espacios de encuentros a fin de poder llegar cercanamente al terreno de los pequeños productores de la localidad, trabajando en sus fincas, así como también se realizaron encuentros teóricos prácticos en instalaciones de la EFA 141Fortaleza Campesina, la Escuela 788 Colonia Lote 90 y del CRES en General San Martin Chaco. En los mismos participaron estudiantes y docentes de FCV-FCA guiando e interactuando con los distintos actores involucrados (Figura 6).

Se pudieron poner en común las experiencias de prácticas de manejo culturizadas, así como también se analizaron los aportes a la salud de la incorporación de buenas prácticas de manejo sanitario en la prevención de las zoonosis. Se observaron las necesidades de los pequeños productores en el acompañamiento de las instituciones sanitarias locales. Los encuentros propiciaron el intercambio de experiencias entre productores compartiendo las potencialidades y debilidades por las que atraviesan y generando en conjunto alternativas de trabajo que favorezcan prácticas de manejo sustentables y seguras.

Además, en cada encuentro se difundió material impreso con medidas de educación sanitaria de las principales zoonosis regionales, distribuido por familia, alumnos y docentes de la EFA. Al mismo tiempo, se generaron temáticas disparadoras para la sanidad humana y animal desde el rol comunitario en general.

Todo lo aportado a partir de estas experiencias, se pudo trasladar a la comunidad académica a través de presentaciones en encuentros científicos y técnicos que permitieron que los estudiantes hagan visible el rol extensionista de la universidad en los ámbitos regionales.

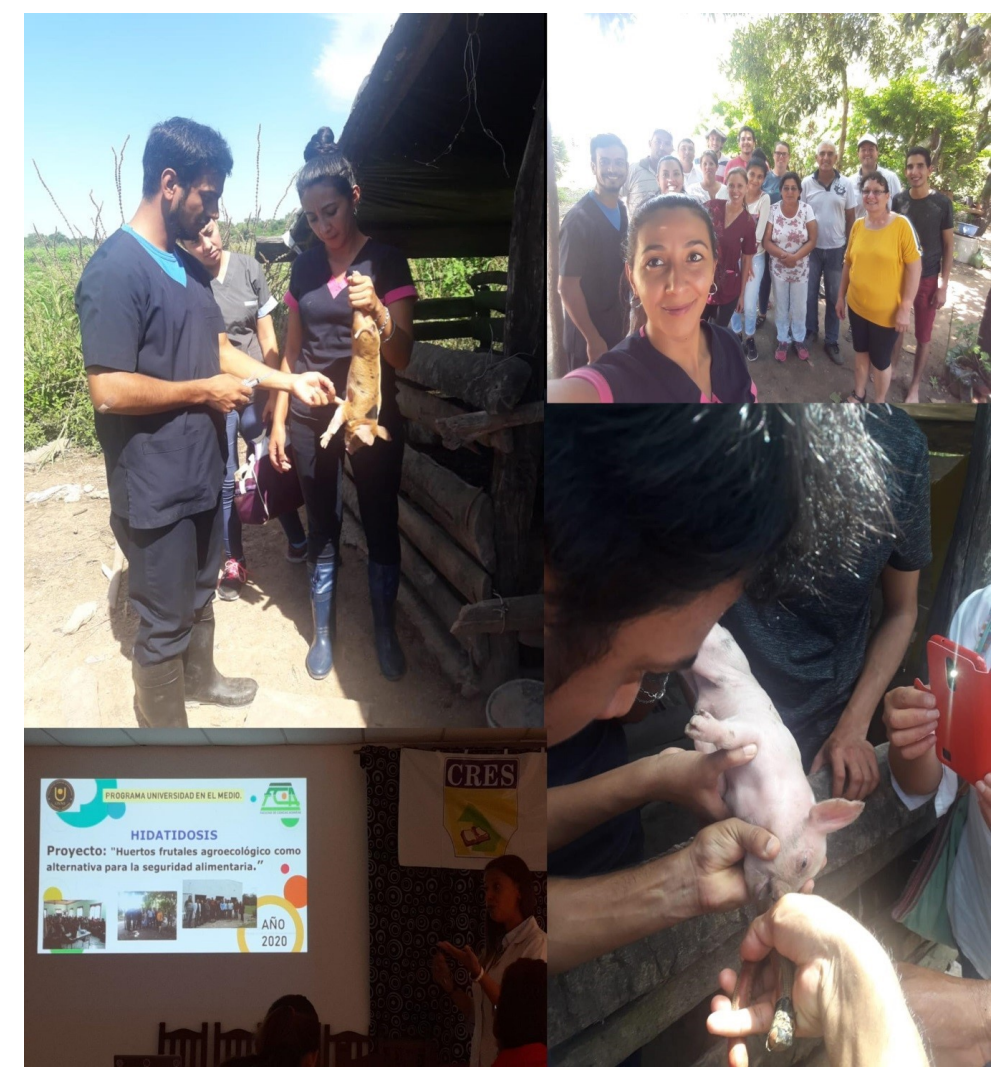

Figura 6. Sensibilización y prevención de la zoonosis - Experiencias de prácticas de manejo culturizadas y análisis de los aportes a la salud de la incorporación de buenas prácticas de manejo sanitario en la prevención de las zoonosis.

\section{Participación en ferias}

Se participó con material vegetal de ananá y plantines de mamón en la feria de pequeños productores de Mi Chacra. En el mismo se presentaron y discutieron experiencias con los asistentes respecto a estos cultivos y se propició un espacio de intercambio de material vegetal (Figura 7). Se promocionó la economía comunitaria con las ventas e intercambio de especies entre vecinos. Los productos excedentes del autoconsumo de sus huertos frutales se pueden destinar para la venta de frutos frescos y a menor costo a la comunidad beneficiando a más familias de la localidad. 


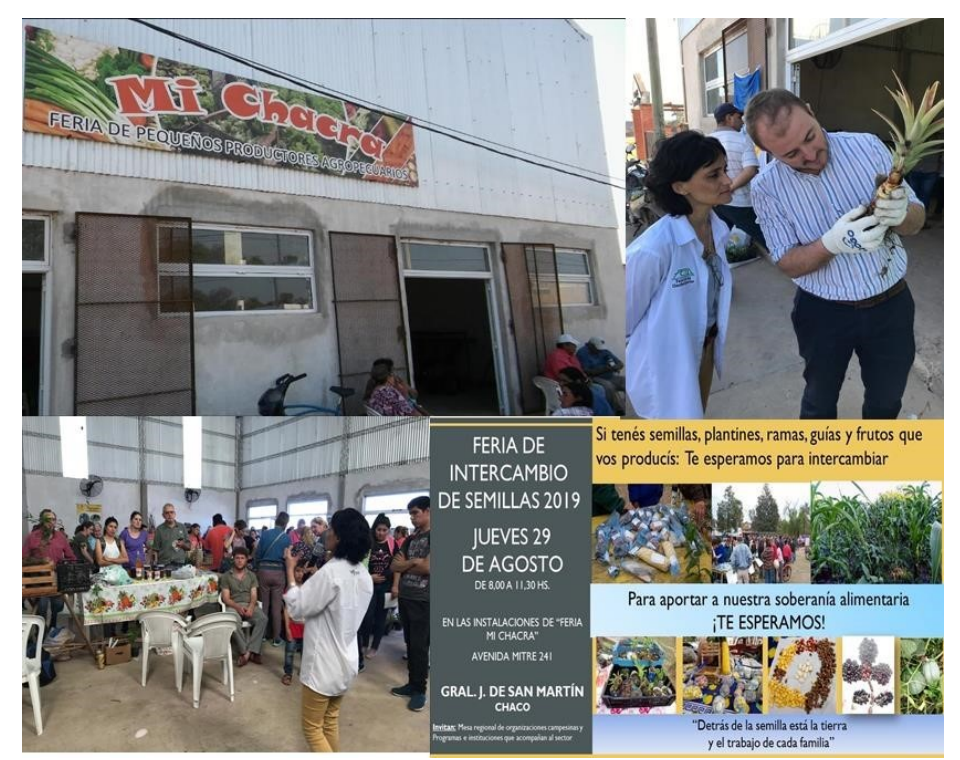

Figura 7. Participación en la feria de pequeños productores de Mi Chacra General San Martín Chaco.

\section{CONSIDERACIONES FINALES}

El desarrollo de este proyecto ofreció a las familias, pequeños productores y comunidad de San Martín Chaco aspectos prácticos y valiosos de los huertos frutales para fortalecer su seguridad alimentaria. La diversidad de información y de material vegetal frutícola, contribuyó a la promoción de la economía comunitaria con las ventas e intercambio de especies entre vecinos.

Los encuentros propiciaron el intercambio de experiencias entre alumnos, productores y comunidad en general, compartiendo las potencialidades y debilidades por las que atraviesan, generando en conjunto alternativas de trabajo que favorezcan prácticas de manejo sustentables y seguras.

Para nuestra comunidad Universitaria fue un aporte de experiencias en lo que se refiere al trabajo en grupos interdisciplinarios, que potencia en los alumnos el profesional extensionista con sensibilidad social.

\section{AGRADECIMIENTOS}

\section{Participantes del proyecto}

Docentes y Alumnos de FCA y FCV UNNE: Ing. Agr. Alvez, Natalia V.; Zarabozo Gamarra, Leisa M.; López, Duilio A.; Luxen, Maira E.; Ramírez, Marina A.; Quiroga, Joaquín; Chequín, Maximiliano; Martínez Dimaria, Gustavo A.; Basualdo, Lucia S.; Romero, Gustavo Pablo; Segovia, Carlos D., Ojeda, Maira;

Participantes Instituciones contrapartes: Med. Vet. Bianchi, Facundo O.; Com. Social Ramos, Dora; Profesor Ivanoff, Rodolfo; Ing. Agr. Piamontese, Elena; Ing. Agr. Monfardini, Joana.

Instituciones: Facultad de Ciencias Agrarias y Facultad de Ciencias Veterinarias de la Universidad Nacional del Nordeste. UEGP N ${ }^{\circ}$ 141: EFA 141 Fortaleza Campesina; INCUPO; Municipalidad de General San Martin Chaco.

\section{Financiación}

Las acciones de este trabajo fueron financiadas y realizadas en el marco del Programa "Universidad en el medio" Resolución 290/18 y 194/19 C.S.

\section{BIBLIOGRAFÍA}

FAO (2011) Seguridad Alimentaria Nutricional, Conceptos Básicos. Disponible en: http://www.fao.org/3/aat772s.pdf

Organización Mundial de la Salud. Ginebra (2003) Dieta, nutrición y prevención de enfermedades crónicas. Disponible en: https://www.who.int/nutrition/publications/obesity/WHO_TRS_916_spa.pdf 